\title{
Efficiency of Executive Function: A Two-Generation Cross-Cultural Comparison of Samples from Hong Kong and the United Kingdom
}

\author{
Michelle R. Ellefson \\ Faculty of Education, University of Cambridge \\ Florrie Fei-Yin $\mathrm{Ng}$ \\ Department of Educational Psychology, Chinese University of Hong Kong \\ Qian Wang \\ Department of Psychology, Chinese University of Hong Kong \\ Claire Hughes \\ Centre for Family Research, University of Cambridge
}

This manuscript was accepted for publication $1^{\text {st }}$ November 2016 by Psychological Science. This is the version submitted on $31^{\text {st }}$ October 2016 (it was the second revision).

Correspondence concerning this article should be addressed to Michelle R. Ellefson, University of Cambridge Faculty of Education, 184 Hills Road, Cambridge, CB2 8PQ, United Kingdom. Email:mre33@cam.ac.uk. 


\begin{abstract}
While Asian preschoolers acquire executive functions (EFs) earlier than their Western counterparts (e.g., Sabbagh, Xu, Carlson, Moses \& Lee 2006), little is known about whether this advantage persists into later childhood and adulthood. Addressing this gap, the current study presented four computerized EF tasks (providing measures of inhibition, working memory, cognitive flexibility and planning $)$ to a large sample $(n=1,427)$ of 9 - to 16 -year-olds and their parents living in the United Kingdom and in Hong Kong. Highlighting the importance of combining developmental and cultural perspectives, our findings showed both similarities and contrasts across sites. Specifically, adult EF performance did not differ across sites and agerelated changes in EF for both children and parents as well as a modest intergenerational correlation appeared culturally invariant. In contrast, school-aged children and young adolescents in Hong Kong outperformed their UK counterparts on all four EF tasks, consistent with previous findings from preschool children.
\end{abstract}

Keywords: Executive Functions, Cross-cultural Research, Inhibition, Working Memory, Cognitive Flexibility, Planning. 


\section{Efficiency of Executive Function: A Two-Generation Cross-Cultural Comparison of Samples from Hong Kong and the United Kingdom}

Executive functions (EFs), defined as the set of higher-order cognitive processes that underpin flexible, goal-directed action and adaptive responses to novel or complex situations (e.g., Hughes, Ensor, Wilson \& Graham, 2010), have attracted remarkable interest from both cognitive and developmental psychologists. For example, meta-analytic reviews have shown that variation in EFs are associated with individual differences in externalizing problems (Astill, van der Heijden, van IJzendoorn, \& van Someren, 2012; Schoemaker, Mulder, \& Deković, 2013), theory of mind (Devine \& Hughes, 2013), numeracy (Bull \& Lee, 2014), and literacy (Kudo, Lussier, \& Swanson, 2015). Alongside this work other researchers have identified parental influences on EF skills and examined the ways social factors can influence EF development. For example, there are intergenerational correlations in EF skills (Cuevas, DeaterDeckard, Kim-Spoon, Wang, Morasch, \& Bell, 2014) as well as beneficial effects of parental scaffolding (for a review see Hughes, Roman, \& Ensor, 2014), attachment relationships (Bernier, Beauchamp, Carlson, \& Lalonde, 2015) and bilingualism (Bialystok, 2015). Conversely, other studies (often involving clinical samples) indicate adverse effects of family chaos (Brown, Ackerman, \& Moore, 2013), maltreatment (for a review see Belsky \& de Haan, 2011) and exposure to maternal depression (Hughes, Roman, Hart, \& Ensor, 2013).

Family influences do not, however, operate within a vacuum and so cultural influences on children's EFs also deserve attention. One striking and consistent finding is that preschoolers from Asian countries typically do better on EF tasks than their Western counterparts (e.g., Lewis, Koyasu, Oh, Ogawa, Short, \& Huang, 2009; Sabbagh, Xu, Carlson, Moses \& Lee, 2006). This contrast has been interpreted as reflecting differences in socialization goals and practices, with Asian children being taught the importance of self-control from a very early age. To date, crosscultural comparisons of EF have largely been restricted to preschoolers and framed by a 
separate literature that suggests this East-West difference is specific to EF and does not extend to related cognitive skills, such as theory of mind. Extending the developmental scope of this research to pre-adolescence, Wang, Devine, Wong and Hughes (2016) reported an advantage in EF for children from Hong Kong (HK) relative to their peers in the United Kingdom (UK) in two separate studies involving HK children attending local or international schools. Interestingly, the same two study samples showed an advantage in the opposite direction for theory of mind, although this depended on school type: UK children outperformed HK children attending local schools but performed similarly to HK children attending (UK style) international schools. In other words, while general cultural differences appear to contribute to geographical contrasts in EF, pedagogical experiences appear particularly salient for children's developing concepts of mind (see also Hughes, Devine, Ensor, Koyasu, Mizokawa, \& Lecce, 2014).

In another cross-cultural study that deserves note, Imada, Carlson and Itakura, (2013) found that 4- to 9-year-old children from Japan outperformed their counterparts from the USA on tests of both EF and context-sensitivity; moreover, the group difference in context sensitivity fully explained the contrast in EF. In discussing these findings, these authors drew on adult studies demonstrating a contrast between holistic, global thinking styles and analytic, local styles of information processing that mirror the philosophical legacies of Ancient China and Ancient Greece (e.g., Nisbett, Choi, Peng, \& Norenzayan, 2001). However, this context-sensitivity account of group differences in EF is challenged by recent re-analyses of a previously reported Chinese advantage in perspective taking (Wu \& Keysar, 2007). Specifically, by applying timeseries analyses to eye-tracking data, Wu, Barr, Gann and Keysar (2013) showed that the group contrast emerged very late in processing, indicating a contrast in top-down suppression rather than in integration of knowledge (i.e., in EF rather than in context sensitivity). That said, as the authors acknowledge, without direct measures of EF it cannot be concluded that the 'East-West' contrast in EF extends beyond childhood (Wang et al., 2016). We hypothesize that the Asian 
advantage in EF extends into late childhood $(\mathrm{H} 1)$ and adulthood $(\mathrm{H} 2)$ and that EF scores on each task will be correlated across child-parent dyads (H3).

Finally, it is worth noting that cultural influences are dynamic rather than static: in a rapidly changing world, one might expect 'cohort effects', such that any between-site contrasts may therefore differ across generations in magnitude and/or nature. The current cross-cultural study of EF is, to our knowledge, the first to adopt an intergenerational design. Previous studies have found correlations between parents and children from within the same culture group, but we don't yet know if that is consistent across cultures. Two further strengths of this study deserve note. First, exactly the same EF tasks were administered to parents and children, enabling parent-child comparisons to be made for the first time. Second, a computerized battery was adopted that enabled testing to be conducted in whole class. As a result, our study sample is much larger than in previous studies, increasing the reliability of our findings and enabling us to compare, for the first time, age-related changes in EF within each cultural group. 


\section{Method}

\section{Participants}

In total, participants in this study included 886 children and 541 parents (additional demographic details in Table 1). From this overall sample, a total of 540 full parent-child dyads were available for family analyses. This sample was recruited from state and private HK schools, but only state schools in the UK. The pattern of recruitment is expected given that the proportion of school children from these ages attending private (i.e., with fees/payments for enrollment) schools in $\mathrm{HK}$ is about $22 \%$ and about $7 \%$ in the UK. The contrasting sample size for children and parents in the UK reflected difficulties in recruiting parents for children attending schools in lower-income areas. It is also worth noting that the UK dyad sample included 23 pairs of siblings; for most of these, data were available from both parents, enabling us to create separate parentchild dyad pairs. The ethics committees from all universities involved in this project reviewed and approved this research project. All parents provided written consent and the children verbal assent. Families in the UK were given $£ 20$ and children received small prizes for taking part. Families in $\mathrm{HK}$ were given $\mathrm{HK} \$ 300$ and children received small prizes for taking part, except in one school that did not want to offer families any incentives. All schools were also provided a gift for their participation.

Relatively few participants had home language backgrounds that differed from the main school language. In particular, only $15 \mathrm{HK}$ children spoke a language other than Cantonese at home. That said, HK children receive English lessons from their first year of schooling. In the UK sample, 43 children did not speak English at home. These children spoke a diverse set of languages that represented the six habitable continents.

Missing data. All but 8 of the 840 children included for analyses completed all the EF tasks (the 8 with incomplete EF data were all from the UK). There was also missing demographic data from 16 HK parents, 25 UK parents and 37 UK children. 


\section{Table 1.}

Demographic details for the participants.

\begin{tabular}{|c|c|c|c|c|}
\hline & \multicolumn{2}{|c|}{ Hong Kong } & \multicolumn{2}{|c|}{ United Kingdom } \\
\hline & Children & Parents & Children & Parents \\
\hline$N$ & 371 & 266 & 516 & 275 \\
\hline Age (years $\left.{ }^{1}\right)$ & $12.21(0.99)$ & $43.84(5.22)$ & $11.91(0.93)$ & $44.06(4.78)$ \\
\hline \multicolumn{5}{|l|}{ Gender ${ }^{2}$} \\
\hline females & 174 & 189 & 242 & 186 \\
\hline males & 197 & 70 & 245 & 64 \\
\hline Additional Languages & \multicolumn{2}{|c|}{15} & \multicolumn{2}{|c|}{43} \\
\hline $\begin{array}{l}\text { Formal Education } \\
\text { (years) }\end{array}$ & $7.21(0.83)$ & $14.43^{3}(3.59)$ & $7.68(0.89)$ & $17.36^{3}(3.03)$ \\
\hline Total Dyads & \multicolumn{2}{|c|}{262} & \multicolumn{2}{|c|}{$278^{4}$} \\
\hline
\end{tabular}

Notes

${ }^{1}$ Standard Deviations in parentheses

${ }^{2}$ Not all participants disclosed their gender

${ }^{3}$ Where the international equivalent of completing secondary education (or just before starting university) is 14 years, a bachelor's degree 18 years, master's degree 20 years and doctoral degree 22 years

${ }^{4}$ The dyads total is slightly larger than the number of parents for the UK due to having families where both parents and/or multiple children participated in the study

\section{Design}

Our overall design included two between-subject factors: site (UK or HK) and generation (parent or child). In both sites, parents and children completed the same EF tasks, enabling direct comparisons of task performance. To avoid the complications presented by firewalls, the same program was installed on a UK server and a HK server administered from the same secured website. The (very limited) verbal instructions for the tasks were translated into Chinese 
for HK participants and participants completed all four EF tasks during one session. Task measures included both accuracy and reaction time (RT) data across multiple trials, enabling us to account for speed-accuracy trade-offs by using efficiency scores, calculated as:

$$
\text { Efficiency }=\frac{\text { Total Number Correct }}{\text { Mean RT to Correct Trials }}
$$

Additional variables and data stopping procedures. This work presents findings that indicate differences in EF across sites and generations. The framing study also includes questions about family influences on and the educational impact of EF; the results relating to these additional questions will be reported separately for reasons of space and coherence. Supplementary results include trial-by-trial data within each of the EF tasks. As outlined in the grant application to fund this research project, we aimed to collect data from 300 parent-child dyads from each site to enable enough statistical power to run either hierarchical regressions or structural equation models using the full dataset or subsets. This target was nearly reached $(n=$ 590) but some dyads were removed from the present analyses because one partner had not completed the EF task battery.

\section{Materials and Procedures}

We used an existing secured site, Thinking Games (see http://instructlab.educ.cam.ac.uk/TGsummary/) for more details and example stimulus screens) to administer the EF tasks in our EF battery. Participants completed the tasks in varied orders with breaks between tasks if needed. Generally, children completed the EF task battery at school (during whole-class sessions) and parents completed the tasks at home. However, some families from both sites chose to complete the tasks in a university lab. Participants were encouraged to respond as quickly as possible while still being accurate. 
Inhibition - The Stop Signal Task. This child-friendly version of the original Stop Signal task (Logan, 1994) includes an image of a soccer field with the ball centrally positioned either on the left- or right-hand side of the screen. For each of 108 trials (presented in three blocks), participants are instructed to click the left arrow key when the soccer ball is on the left-hand side of the screen (54 trials) and the right arrow key when the soccer ball is on the right-hand side of the screen (54 trials), but to refrain from clicking when they hear the referee's whistle, which is played randomly on $20 \%$ of the trials, also called No Go trials. Following standard stop signal procedures, the gap between the presentation of the picture and the presentation of the whistle is increased or decreased depending on participant accuracy. The first whistle is played 250 msec after the picture appeared. If the participant successfully inhibits a response, then the whistle is played $50 \mathrm{msec}$ later during the next Stop trial. If they do not successfully inhibit, then the picture is played $50 \mathrm{msec}$ sooner on the next trial.

Working Memory - A Spatial Span Task. This modification of the Corsi blocks tasks (Corsi, 1972) is divided into two parts: forwards (presented first) and backwards. On each trial, the screen display includes an array of 9 boxes, some of which light up in a pre-selected order. Participants are asked to click on the boxes either in the same order (forwards) or the reverse order (backwards). After two practice items (each with 2 boxes lighting up), participants receive sets of increasing length, completing two each of 3, 4, 5, 6 and 7 items sequences for the forwards and backwards items and additional sequences of 8 and 9 items for the forwards items only. Excluding the practice trials, there is a possible total of 16 forward sequences and 12 backward sequences, but testing automatically discontinues after five consecutively incorrect trials.

Shifting - The Figure Matching Task. This task is a slightly modified presentation of Ellefson, Shapiro \& Chater (2006) and included 128 trials, each with four simultaneous events. A target figure in the center of the screen varies by shape (triangle or circle) and/or color (blue or red). The top of the screen displays an instruction to sort by shape or color, by pressing one of 
two keys on the computer keyboard that corresponded to the location of two smaller figures displayed in the lower corners of the screen matching the target in either dimension (shape or color).

The trials were presented randomly within four 32-trial blocks (counter-balanced between participants): two pure blocks (either all color trials or all shape trials) and two mixed blocks with color and shape trials presented using an alternating-runs sequencing (Rogers \& Monsell, 1995) that change tasks every two trials (i.e., color-color-shape-shape-color-color-shape-shape-etc.). One of these mixed trials began with a color trial and the other mixed block began with a shape trial (again, this was counterbalanced across participants). There are thus two trial types: repeat and switch. In Repeat trials (included in both pure and mixed blocks) participants continue the same task as the previous trial. In the Switch trials (mixed block only), participants changed to a different task from the previous trial.

Planning - The Tower of Hanoi Task. This is a computerized version of the task used by Welsh (1991). Participants see two arrangements of disks on the screen and are invited to arrange the disks in the bottom set to match the top set in as few moves as possible and without placing a larger disk on a smaller disk. The minimum number of moves needed to transform the bottom set to match the top set increased with each successful matching. The increased number of minimum moves increased the difficulty of the task.

After a practice 2-move 3-disk problem (with feedback for illegal moves) participants are given six more 3-disk problems, including 2-, 3-, 4-, 5-, 6-, and 7-move problems. This is followed by three 4-disk problems, including 7-, 11-, and 15-move problems. If participants erroneously placed a larger disk on a smaller disk, they are given a reminder message that their move was not allowed. This message stayed up for $2000 \mathrm{msec}$. The disk is then returned to its original location, with that illegal move counting as one move. To continue onto the more difficult problems, participants need to make two consecutive minimum-move solutions. On each problem, participants have a maximum of 20 moves to match the goal arrangement before being 
offered a new attempt (with a maximum of six attempts to achieve two consecutive minimummove solutions). The task ends when participants have either successfully solved all problems within these constraints or when they reached a problem that they could not solve twice in a row within six attempts.

\section{Data Processing and Analyses}

Overall accuracy and RTs to correct trials were used to create efficiency scores for each of the EF tasks using Equation 1. Next, efficiency z-scores were calculated individually for each task. Standardizations were generated using all participants. Finally, individual participants zscores from each EF task were averaged together to create a standardized EF efficiency aggregate score. We chose this standardized EF aggregate over factor scores both to facilitate comparisons with another intergenerational study of EF (Cuevas et al., 2014) and because factor solutions were different for children and adults in this dataset and in previous work (e.g., Miyake, Friedman, Emerson, Witzki, Howerter \& Wager, 2000; Wiebe et al., 2008). As a precaution, we verified our findings with analyses using factor scores and found no change in the pattern of results.

Standardized EF efficiency aggregate scores were analyzed using a $2 \times 2$ ANOVA with the between-subjects factors of site (HK, UK) and generation (child, parent). We focus on efficiency scores because it helps us account for both accuracy and response speed and because when analyzed separately accuracy and response speed can show different patterns with adults and children. More specifically, previous studies with adults in these types of tasks commonly show ceiling effects for accuracy (e.g., Logan, 1994; Miyake et al., 2000; Rogers \& Monsell, 1995). whereas with children generate a wider range of accuracy scores (e.g., Akshoomoff et al., 2014; Astill et al., 2012). Age-related improvements for children in accuracy tend to positively correlate with age-related improvements in RT, but the relationship between accuracy and RT is not the same during middle adulthood where accuracy holds steady and RT performance declines (e.g., Reimers \& Maylor, 2005). Efficiency scores account for the various problems of exploring 
accuracy and RT independently, while affording group comparisons in a single analysis. In addition, the instructions to participants, as outlined in the method section, were that they respond as quickly as they could while still being accurate, correspond directly to an analysis using efficiency scores.

However, efficiency scores can mask response patterns. There could be differences in response strategies across the generations and sites and efficiency scores might not be a true reflection of the underlying accuracy and RTs, To investigate these ideas, we follow up our initial analysis with similar ANOVAs that use standardized aggregate z-scores for accuracy and RTs to correct trials as dependent variables. The standardized aggregates for accuracy and RT were calculated using the same procedures as the standardized EF efficiency aggregate score. To make sure that the standardized aggregate scores were not biased by one or more of the individual EF tasks, we ran the same ANOVAs described above separately for each EF task using the standardized scores for that EF task as the dependent variable.

Several verification checks were conducted to eliminate the potential contribution of various biases on the main findings. We evaluated the influence that two core demographic variables of age and education might have on the overall findings by conducting a $2 \times 2$ ANCOVA with the same between-subject factors (site and generation) and age as a covariate. Where appropriate, significant effects and interactions were followed-up using Tukey's post-hoc test to control for Type I error. Effect sizes were calculated using Partial Eta-Squared $\left(\eta_{p}{ }^{2}\right)$. Our child sample provided $80 \%$ power to detect a small effect size $\left(\eta_{p}{ }^{2}=.01\right)$ and the parent sample $80 \%$ power to detect a medium effect size $\left(\eta_{p}{ }^{2}=.09\right)$, substantially reducing the risk of Type II error. 


\section{Results}

In this results section we first examine site (UK-HK) and generation (parent-child) effects (and their interplay) on EF performance using overall task performance and within-task contrasts in participants' performance using performance cost metrics. Capitalizing on the large sample size for the current study, we then examine the relationship between age and EF in each generation. Finally, building on this study's two-generation design we examine the association between parents and children's EF performance (both overall and within each site).

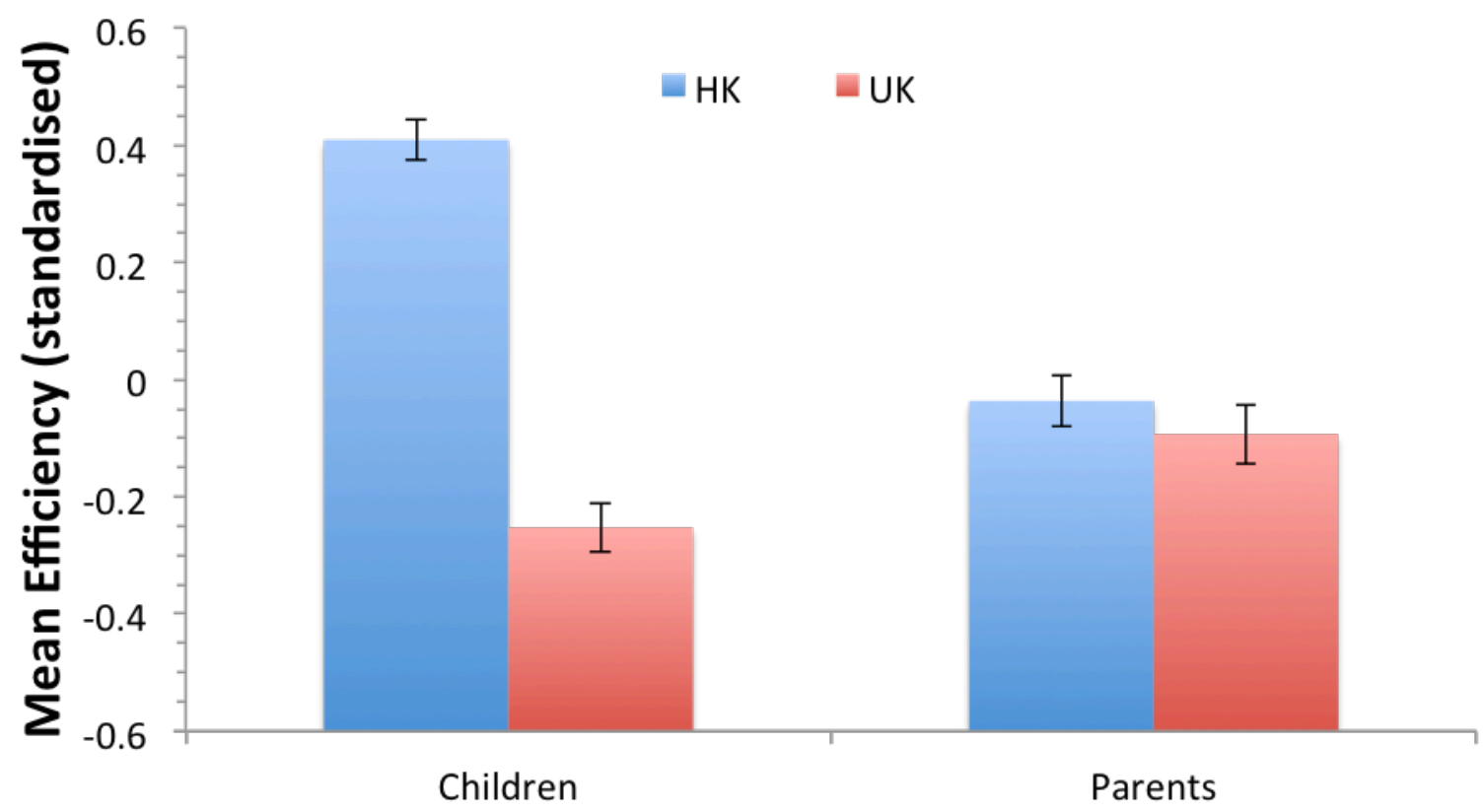

\section{Generation}

Figure 1. Mean EF aggregate z-score for efficiency by site (HK, UK) and generation (child, parent), with the standard error of the mean shown represented by the error bars.

\section{EF Scores Differ by Site and Generation}

As displayed in Figure 1, the mean z-scores for efficiency of overall EF task performance showed significant effects for both sites, $F(1,1423)=101.92, p<.001, \eta_{p}{ }^{2}=.07(\mathrm{HK}>\mathrm{UK})$ and 
generation, $F(1,1423)=16.20, p<.001, \eta_{p}{ }^{2}=.01$ (Children $>$ Parents). There was also a significant interaction between these two factors, $F(1,1423)=72.45, p<.001, \eta_{p}{ }^{2}=.05$, indicating that the UK-HK contrast was evident for children but not parents. On average, HK children performed as well at age 10 as their UK counterparts at age 12 with this two-year lag appearing across the age-span of the study sample. These findings extend the developmental scope of findings from previous studies in which Asian preschoolers show better EF skills than their Western counterparts, our results indicate that in middle childhood and early adolescence children in HK outperform their UK peers - but this effect was not evident for the parent participants in this study.

The results for the aggregate efficiency score were corroborated by similar analyses conducted on aggregate of accuracy and RTs to correct trials. For accuracy, there was a significant effect of generation, $F(1,1423)=187.86, p<.001, \eta_{p}{ }^{2}=.12$ (Parents $>$ Children), but the effect of site was not significant, $F(1,1423)=0.01, p=.90, \eta_{p}{ }^{2}=.00$. However, there was a significant interaction between site and generation, $F(1,1423)=26.15, p<.001, \eta_{p}{ }^{2}=.02$. Follow-up tests indicated that UK parents had the highest accuracy, followed by HK parents, then HK children, and with UK children having the lowest accuracy scores. For RTs to correct trials, there were significant effects of both site, $F(1,1422)=43.07, p<.001, \eta_{p}{ }^{2}=.03(\mathrm{HK}$ faster than UK), and generation, $F(1,1422)=141.66, p<.001, \eta_{p}{ }^{2}=.09$ (Children faster than Parents). In addition, the site by generation interaction was significant, $F(1,1422)=31.75, p<$ $.001, \eta_{p}{ }^{2}=.02$, with HK children having the fastest RTs to correct trials, followed by UK children, who were faster than both HK parents and UK parents.

Taken together, the aggregate efficiency score reflected the HK children giving very fast, correct responses, showing performance advantages over the UK children on both accuracy and response speed. Both groups of parents were more accurate than both groups of children, but this was paired with a slow response speed. The UK parents were significantly more accurate than the HK parents, but they did not have a significantly higher efficiency score because they 
had a slower response speed than the others. This response speed was not significantly slower than the HK parents, but reduced the differences between the HK and UK parents in the efficiency score.

\section{Table 2.}

Correlations between the four EF tasks for parents and controlling for age and education.

\begin{tabular}{|c|c|c|c|c|}
\hline EF Task & $\underline{1}$ & $\underline{2}$ & $\underline{3}$ & $\underline{4}$ \\
\hline \multicolumn{5}{|l|}{ Full Sample } \\
\hline \multicolumn{5}{|l|}{ 1. Inhibition } \\
\hline 2. Working Memory & $.25^{\star \star *}$ & & & \\
\hline 3. Shifting & $.43^{* * *}$ & $.37^{* * *}$ & & \\
\hline 4. Planning & $.18^{* * *}$ & $.31^{* * *}$ & $.29^{* \star *}$ & \\
\hline 5. Composite & $.68^{* \star *}$ & $.71^{* * *}$ & $.77^{\star \star *}$ & $.63^{* * *}$ \\
\hline \multicolumn{5}{|l|}{ Children } \\
\hline \multicolumn{5}{|l|}{ 1. Inhibition } \\
\hline 2. Working Memory & $.27^{* * *}$ & & & \\
\hline 3. Shifting & $.42^{* * *}$ & $.34^{* * *}$ & & \\
\hline 4. Planning & $.16^{* \star *}$ & $.26^{* * *}$ & $.23^{* * *}$ & \\
\hline 5. Composite & $.70^{* * *}$ & $.70^{* * *}$ & $.73^{* * *}$ & $.59^{* * *}$ \\
\hline \multicolumn{5}{|l|}{ Parents } \\
\hline \multicolumn{5}{|l|}{ 1. Inhibition } \\
\hline 2. Working Memory & $.12^{* *}$ & & & \\
\hline 3. Shifting & $.38^{* * *}$ & $.30^{* * *}$ & & \\
\hline 4. Planning & $.15^{* * *}$ & $.29^{* * *}$ & $.26^{* * *}$ & \\
\hline 5. Composite & $.64^{* * *}$ & $.62^{* * *}$ & $.74^{\star * *}$ & $.65^{* * *}$ \\
\hline
\end{tabular}

This pattern of overall performance is replicated for the individual EF tasks and is supported by the correlations between the tasks. As shown in Table 2, scores on the four EF tasks showed consistent correlations with each other for the full sample, ranging from .19 and 
.41 and showing good internal consistency. However, the correlational patterns are not the same across the two generations and sites. In addition, the associations between these composite EF scores and children's non-verbal IQ (indexed by Ravens Progressive Matrices scores) were .34 $(\mathrm{HK})$ and $.40(\mathrm{UK})$, with the difference in magnitude being similar across the two children's samples $(z=1.02, p=.31)$.

Verification checks. The children and parents in this study were from the same family, and as such, generation might not be a fully independent variable. We fully replicated results with additional analyses treating generation as a within-subjects variable. There were a larger number of children in the UK sample whose parents did not complete the EF tasks. To test for potential biases in the data, the analyses were rerun using only instances where we had both children's and parents' data. The results for efficiency and RTs were the same. Although the overall effects and interactions were the same for accuracy, the post-hoc tests were slightly different with gap between the HK and UK children narrowing and no longer significant.

\section{Age and Education Show a Similar Association with EF for HK and UK Samples}

Age. The ages across the sites and generations are not identical, raising the possibility that the results across site can be accounted for by age differences. Against this hypothesis, a 2 (site) $\times 2$ generation ANOVA on participant ages indicated only a significant main effect of generation, $F(1,1345)=32293.64, p<.001$. The main effect of site, $F(1,1345)=0.05, p=.82$, and the site by generation interaction were not significant, $F(1,1345)=2.15, p=.14$, confirming that the child and parental ages were not different across the two sites. 


\section{Children}
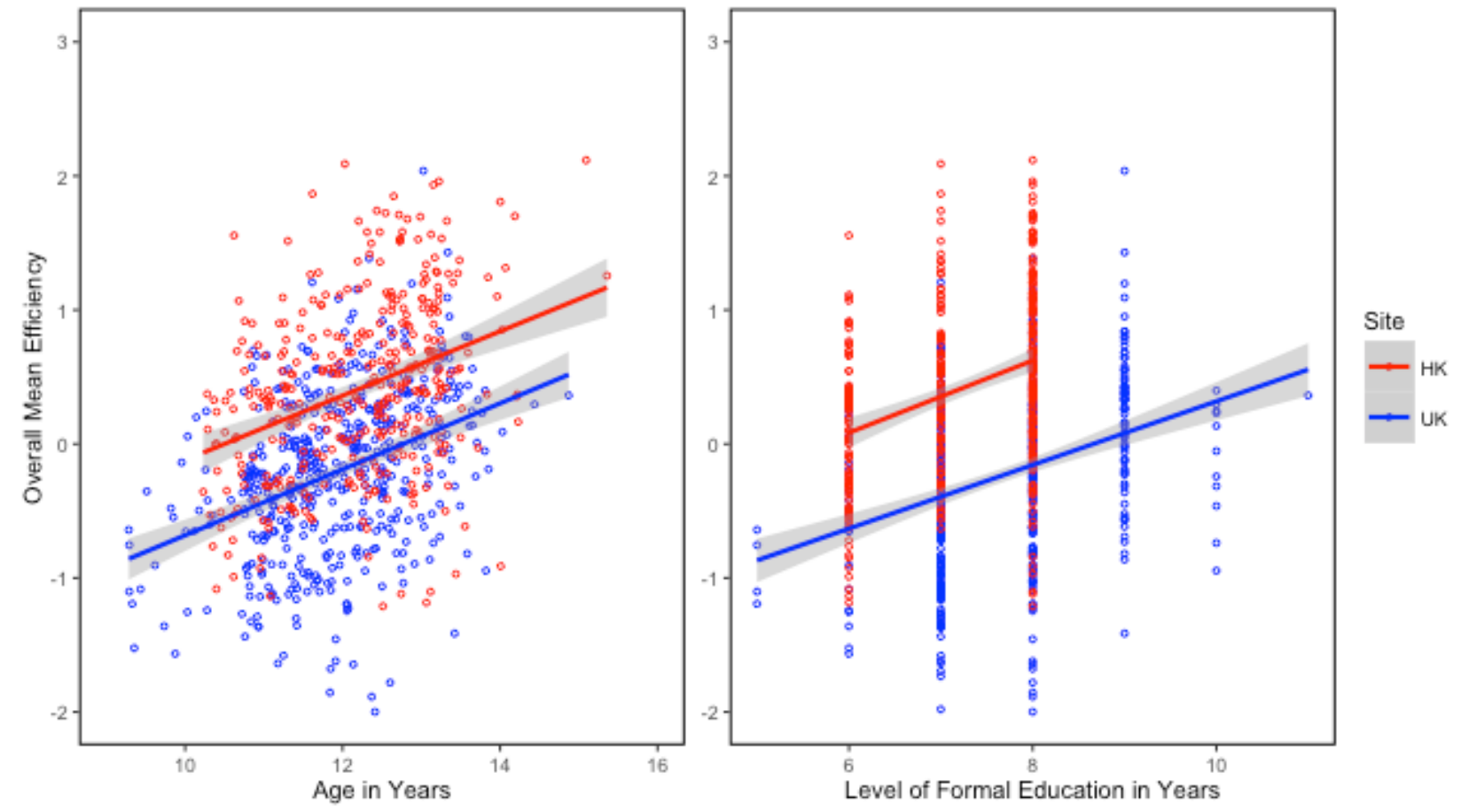

\section{Parents}
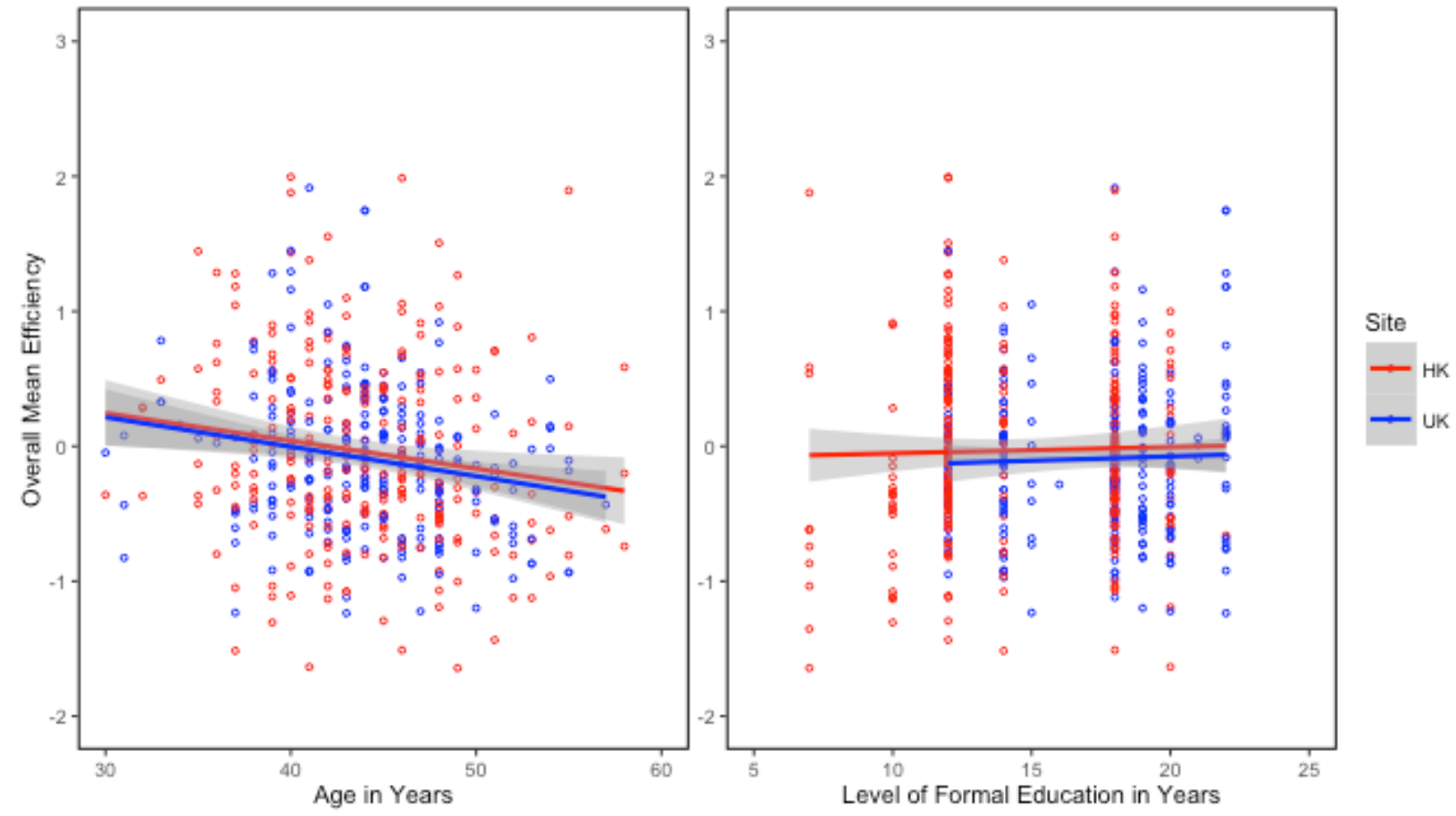

Figure 2. Scatter plots of age and education level by mean EF z-score aggregate for each site (HK, UK) and generation (child, parent). 
The site $\mathrm{x}$ generation interaction effect reported for EF efficiency raises the possibility that UK children eventually catch up with their HK peers. Against this hypothesis, however, scatterplots (Figure 2) indicated a similar influence of age on EF performance for both groups of children, $R_{H K}^{2}=.12, F(1,371)=50.51 p_{H K}<.001, R_{U K}^{2}=.14, F(1,478)=76.98, p_{U K}<.001$. The slopes of age-related changes are the same, but the intercept is higher for the HK children, $Y_{H K}=-2.43+0.23 X_{H K}, Y_{U K}=-3.17+0.25 X_{U K}$. The regression coefficient indicated a similar improvement of about 0.23/0.25 SD for each year of age. As shown in Figure 2, the average EF score at age 10 for HK children was similar to that for UK children at age 12; likewise, the average EF score at age 12 for HK children was similar to that for UK children at age 14 . That is, across the age-span of the children in the current study, there was no evidence of a catch-up effect by early adolescence.

Figure 1 indicated that the parents had a lower efficiency scores than the HK children. Additional analyses indicated that parents had higher accuracy but slower RTs than the children. These results could be driven by slow age-related declines in RT that start in middle adulthood. Supporting this hypothesis, the association between EF efficiency and age was weakly negative, $R_{H K}^{2}=.02, F(1,250)=6.07, p_{H K}=.01, R_{U K}^{2}=.04, F(1,248)=9.54, p_{U K}=.002$ (see Figure 2) . The slopes for age-related changes in EF were similar across sites, but the HK parents had a slightly lower intercept, $\mathrm{Y}_{\mathrm{HK}}=0.86-0.02 \mathrm{X}_{\mathrm{HK}}, \mathrm{Y}_{\mathrm{UK}}=0.87-0.02 \mathrm{X}_{\mathrm{UK}}$. The differences in regressions were significantly different between the two generations, $F(1,1345)=170.28 p<.001, \eta_{p}^{2}=$ 11.

Verification checks. ANCOVAs using age as a covariate and separate slopes for the two generations as well as ANOVAs using age as a continuous factor replicated the main site and cohort findings. Most importantly, the differences across sites for children's EF efficiency and the similarities across sites for parent EF efficiency remained even when the aggregate EF scores were adjusted for age. 
Education. As with age, we tested whether participant education levels varied across the groups using a 2 (site) $\times 2$ (generation) ANOVA. The results indicated significant main effects of site, $F(1,1345)=202.26, p<.001$, and generation, $F(1,1345)=5059.39, p<.001$, and a significant site by generation interaction, $F(1,1345)=107.06, p<.001$. Post-hoc tests indicated that the UK parents had higher educational levels than HK parents and that UK children had more formal schooling than HK children. The difference for the children is due to the later school starting age in HK. The different levels in educational experience across the two sites raises the possibility that the EF differences were driven by educational experience.

As would be expected, educational experience and age were highly correlated for the children at both sites, $r_{H K}=(371)=.85, p_{H K}<.001, r_{U K}=(478)=.93, p_{U K}<.001$. Given these high correlations, it is unsurprising that educational level was a significant predictor of EF performance, $R_{H K}^{2}=.12, F(1,369)=47.86 p_{H K}<.001, R_{U K}^{2}=.12, F(1,512)=69.16, p_{U K}<.001$ (see Figure 2). The slopes of education-related changes are the same, but the intercept is higher for the HK children, $\mathrm{Y}_{\mathrm{HK}}=-1.53+0.27 \mathrm{X}_{\mathrm{HK}}, \mathrm{Y}_{U K}=-2.19+0.25 \mathrm{X}_{\mathrm{UK}}$. The regression coefficient indicated a similar improvement of about 0.27/0.25 SD for each year of education. As shown in Figure 2, the average EF score at 6 years of formal education in HK children was similar to that for UK children with 9 years of formal education. In sum, across the educational-span of the children in the current study, there was no evidence of a catch-up effect by early adolescence.

The educational level varied across sites for parents in the study. In addition to the UK parents having higher education levels than HK parents, education level and age did not correlate for the HK parents, $r_{H K}=(250)=.03, p_{H K}=.61$, but there was a small, but significant correlation for the UK parents, $r_{U K}=(250)=.20, p_{U K}=.002$, with older UK parents having higher levels of education than younger parents. Somewhat surprisingly, the association between EF and education was not predictive across both sites, $R_{H K}^{2}=.00, F(1,257)=0.15, p_{H K}=.70, R_{U K}^{2}$ $=.00, F(1,248)=0.31, p_{U K}=.58$ (see Figure 2). For the parents, the slopes for age-related changes in EF were similar, $\mathrm{Y}_{\mathrm{HK}}=-0.10+0.005 \mathrm{X}_{\mathrm{HK}}$ and $\mathrm{Y}_{\mathrm{UK}}=-0.20-0.006 \mathrm{X}_{\mathrm{UK}}$. The older UK 
parents had higher education levels, but this educational advantage is not predictive of improved EF task performance. The differences in regressions were significantly different between the two generations, $F(1,1390)=29.45, p<.001, \eta_{p}^{2}=.02$.

It could be that educational level is not predictive for the UK parents because it is the older parents who have higher education levels, suggesting that any advantage of increased education is counteracted by disadvantages of increased age. Hierarchical regressions confirmed this idea. Age was a significantly negative predictor of parents' EF task performance when education level was controlled, but education was not a significant predictor with or without controlling for age.

Finally, given the differences in education level and parental participation across the two sites, we checked whether parental education influenced the children's data. Here, we averaged the education level of the participating parent with the education level they reported for the child's other parent and tested whether this was predictive of EF task performance. The results confirmed that parental education was not a significant predictor of children's EF task performance in both sites, $R_{H K}^{2}=.001, F(1,273)=0.39, p_{H K}=.53, R_{U K}^{2}=.008, F(1,232)=$ 1.76, $p_{U K}=.19$ and the regression slopes for the two sites were similar, $Y_{\mathrm{HK}}=0.44-0.01 \mathrm{X}_{\mathrm{HK}}, \mathrm{Y}_{\mathrm{UK}}$ $=-0.44+0.02 X_{U K}$. Similar findings occur when we use only the education level of the participating parent.

Verification Checks. ANCOVAs using education as a covariate and separate slopes for the two generations as well as ANOVAs using education as a continuous factor replicated the main site and cohort findings. Most importantly, the differences across sites for children's EF task performance and the similarities across sites for parent EF task performance remained even when EF scores were adjusted for education. These findings were replicated when we ran additional ANCOVAs and ANOVAs controlling for both age and education. 


\section{Parent and Child EF Scores Are Moderately Related for HK and UK Samples}

In total, 541 parent-child dyads in this study completed the EF tasks. Overall efficiency zscores across the four EF tasks showed a significant (but small) correlation between parents and children, both overall: $r_{\text {total }}(540)=.20, p<.001$ and for each site: $r_{H K}(262)=.26, p<.001$; $r_{u k}(278)=.14, p=.03$. These correlations remained relatively unchanged when controlling for participant age: $r_{\text {total }}(500)=.21, p<.001 ; r_{H K}(242)=.27, p<.001 ; r_{u k}(254)=.15, p=.02$. The correlations were similar if conducted using accuracy or RT data.

Verification checks. Finally, to investigate whether the typically different formats for data collection between parents (home, unsupervised individual) and children (school, supervised by researchers in large groups) influenced EF task performance, we ran the same correlations for parents and children who had a researcher supervising the data collection and again for instances where parents and children completed the task individually and supervised by the researchers. The results confirmed that the different supervision formats were not an explanation for the findings.

\section{Results Summary}

This study is methodologically innovative in its two-generation design (enabling the integration of developmental and cultural perspectives) and its use of online EF tasks (enabling efficient data-collection from a large sample). The study results can be summarized by three main findings. Across sites, the East-West contrast in EF efficiency was evident in early adolescence, but not in middle adulthood. Second, across both sites child EF efficiency scores increased substantially with age; in contrast, parent EF showed a small and negative association with age. Third, within child-parent dyads, the intergenerational association in EF performance was modest, but significant. 


\section{Discussion}

This study is the first to explore: (1) cross-cultural contrasts in adult EF and (2) the cultural universality of both age-related improvements in EF and parent-child associations in EF. Demonstrating that online EF testing is feasible and valid is a further contribution to the field, particularly as this methodology facilitates the recruitment of large samples that include parents, enabling performance on the same task battery to be compared across generations. By reducing verbal demands, these tasks also minimize the role of the researcher (and attendant biases) and they facilitate standardized testing across different language groups. Building on these methodological innovations, the findings highlight the value of combining cultural and developmental perspectives. Specifically, our results show both a site $\mathrm{x}$ generation interaction for EF and the cultural universality of associations between EF and key participant characteristics (age, education). As such, we extend existing findings that show a clear EastWest contrast in preschool children's EF, with the 6-month difference for preschool children (Sabbagh et al., 2006) expanding to 2 years by late childhood and early adolescence. This contrast may reflect socio-developmental factors (e.g., self-control as a key socialization goal) or educational experiences (e.g., increased bilingualism for HK children). However, given that Chinese adults appear to show better perspective-taking and response control than American adults (Wu \& Keysar, 2007; Wu et al, 2013), our null results for parents is surprising.

A key methodological innovation in this study was the use of an online platform of EF tasks, which enabled detailed task data to be collected from large samples at each site. Given the novelty of this approach, it is reassuring that efficiency scores indicated good internal consistency. Importantly, correlations between individual tasks and between EF aggregate scores and non-verbal IQ were similar in magnitude to those in studies involving one-to-one testing (e.g., Carlson, Mandell \& Williams, 2004; Fitzpatrick, McKinnon, Blair, \& Willoughby, 2014; Wiebe, Espy \& Charak, 2008). Together, these findings suggest that the data gathered from these whole-class sessions are as reliable or valid as individual assessments. 
The online format might, however, have affected the two generations differently, suggesting a possible explanation for the modest association in EF within parent-child dyads. Previous intergenerational work (Cuevas et al., 2014) used manual and computer tasks, but focused primarily on accuracy instead of RTs. Future work should include both manual and computerized tasks and account for both accuracy and RTs and control for the age-related declines in RT performance on EF tasks that begin before middle adulthood (e.g., Reimers \& Maylor, 2005). The work here includes a wider span of ages for the children and parents than reported previously. Participant age does seem to have an impact on EF performance for both parents and children. A more precise exploration of genetic contributions on EF would require a more constrained range of ages for both children and parents. Alternatively, the contrast between the relatively strong intergenerational association in EF in prior research with preschool children (Cuevas et al., 2014) and the weaker results observed here may reflect a genuine waning in parental influences on children's EFs. Existing work has focused heavily on toddlers and preschool children (Hughes, 2011), but children become more independent and spend much less time with their parents by middle to late-childhood, making it possible that other socializing forces eclipse parental influences. Longitudinal data straddling preschool and middle childhood are needed to test this hypothesis.

\section{Theoretical Implications}

How should the divergent results from the cross-cultural comparison for children and parents be explained? One possibility is that the relevant cultural differences are specific to norms regarding children. For example, the emphasis on order and harmony within Confucian cultures means that $\mathrm{HK}$ children receive frequent guidance regarding the need to inhibit individual desires (Tardif, Wang, \& Olson, 2009); this explicit socialization may mean that compliance with collectivist norms requires less effortful control in adulthood. For example, a recent cross-cultural study found that UK parents showed greater awareness of children's desires and interests compared to HK parents (Hughes, Devine \& Wang, 2015). An alternative 
possibility is that the discrepant findings from parents and children reflect the dynamic nature of culture. In particular, a series of educational reforms in $\mathrm{HK}$ in the last two decades has led to major changes in the education system, such that HK children could have different learning experiences from their parents, including heightened pressure for students to achieve in both academic and extra-curricular activities. Additional work is needed to confirm the influence of parental attitudes and changes in the education system as potential explanations of the current findings.

Studies of adult cognition have reported cross-cultural contrasts in attention style or context sensitivity (e.g., Imbo \& Lefevre, 2009; Kuwabara \& Smith, 2012), described metaphorically as the contrast between the wide-angle and zoom lens for a camera (Nisbett et al., 2001). Neurophysiological research also highlights the value of considering context sensitivity and EF in tandem. For example, in a review of changes across adolescence in the neurological and functional maturity of the rostral prefrontal cortex (traditionally viewed as a neural substrate for EF), Dumontheil, Burgess and Blakemore (2008) argued that this region is also critical for relational reasoning. As noted earlier, recent findings from both adults and children also suggest an intriguing overlap between context sensitivity and EF, although the causal direction of this association remains controversial. Specifically, Wu et al. (2014) argue that Chinese adults' superior perspective-taking skills (i.e., context sensitivity) reflects an advantage in the suppression of irrelevant information (i.e., EF). In contrast, Imada et al. (2014) argue that Japanese children's EF development is facilitated by a cultural emphasis on context.

While longitudinal designs are needed to test the above hypotheses, our findings indicate that cultural contrasts may differ in nature as well as magnitude at different points along the lifespan. We aim to increase the scope of this research by examining the correlates of individual differences in EF among the children in this study. To our knowledge, the current study is the first published study of EF to include intergenerational alongside across cultural comparisons. Clearly then, our findings require both independent replication and extension to other cultures. 


\section{Author Contributions}

All authors contributed to the study concept and design as part the grant application funding this research. Testing, data collection and data entry were mainly supervised by M.R. Ellefson (UK site) and F.F. Ng (HK site). M.R. Ellefson performed the data analysis. C. Hughes and M.R. Ellefson interpreted the data and drafted the manuscript, F.F. Ng and Q. Wang providing critical revisions. All authors approved the final version of the manuscript for submission. 


\section{Acknowledgements}

A joint-council award to the authors funded this research (ES/K010225/1: Economic and Social Research Council, Research Grants Council of Hong Kong). Thinking Games website development supported by the Institute of Educational Sciences, U.S. Department of Education, through Grant R305A110932 to the University of Cambridge. The opinions expressed are those of the authors and do not represent the views of the Institute or the U.S. Department of Education. Electronic access to dataset: http://reshare.ukdataservice.ac.uk/851984/.

Thanks to (1) Geoff Martin for Thinking Games website programming; (2) Annabel Amodia-Bidakowska, Jeff Chan, Emma Chatzispyridou, Yiming Han, Joyce Hoi-Ling Ng, Katherine Parkin, Annie Raff, Irene Nga-Lam Sze, Antonia Zachariou for data collection assistance; (3) Rosie Blunt, Hannah Bush, Ying-Kit Chan, Claudia Chu, Shehnaz Dowlet, Ellie Frank, Anton Evans, Yanning Gu, Nelly Hu-Kwo for scoring and data entry assistance; and (4) Richard Parkin for proofreading. 


\section{References}

Akshoomoff, N., Newman, E., Thompson, W.K., McCabe, C., Bloss, C.S., Chang, L., Amaral, D.G., Casey, B.J., et al. (2014). The NIH Toolbox Cognition Battery: Results from a large normative developmental sample (PING)" Neuropsychology, 28, 1-10. DOI: $10.1037 /$ neu0000001

Astill, R.G., van der Heijden, K.B., van IJzendoorn, M.H., \& van Someren, E.J.W. (2012). Sleep, cognition, and behavioral problems in school-age children: A century of research meta-analyzed. Psychological Bulletin, 138, 1109-1138. DOI: 10.1037/A0028204

Belsky, J., \& de Haan, M. (2011). Annual research review: Parenting and children's brain development: the end of the beginning. Journal of Child Psychology and Psychiatry, 52, 409-428. DOI: 10.1111/j.1469-7610.2010.02281.x

Bernier, A., Beauchamp, M.H., Carlson, S.M., \& Lalonde, G. (2015). A secure base from which to regulate: Attachment security in toddlerhood as a predictor of executive functioning at school entry. Developmental Psychology, 51, 1177-1189. DOI: $10.1037 / \operatorname{dev} 0000032$

Bialystok, E., Craik, F.I., Green, D.W., \& Gollan, T.H. (2009). Bilingual minds. Psychological Science in the Public Interest, 10(3), 89-129. DoI: 10.1177/1529100610387084

Brown, E.D., Ackerman, B.P., \& Moore, C.A., (2013). Family adversity and inhibitory control for economically disadvantaged children: Preschool relations and associations with school readiness. Journal of Family Psychology, 27, 443-452. DOI: 10.1037/a0032886

Bull, R., \& Lee, K. (2014). Executive functioning and mathematics achievement. Child 
Development Perspectives, 8, 36-41. DOI: 10.1111/cdep.12059

Carlson, S.M., Mandell, D.J., \& Williams, L. (2004). Executive function and theory of mind: Stability and prediction from ages 2 to 3. Developmental Psychology, 40, 1105-1122. DOI: $10.1037 / 0012-1649.40 .6 .1105$

Clark, C.A.., Sheffield, T.D., Wiebe, S., \& Espy, K.A. (2013). Longitudinal associations between executive control and developing mathematical competence in preschool boys and girls. Child Development, 84, 662-677. DOI: 10.1111/J.1467-8624.2012.01854.X

Corsi, P.M. (1972). Memory and the medial temporal area of the brain. Doctoral Thesis: McGill University.

Cuevas, K., Deater-Deckard, K., Kim-Spoon, J., Wang, Z., Morasch, K.C., \& Bell, M.-A. (2014). A longitudinal intergenerational analysis of executive functions during early childhood. British Journal of Developmental Psychology, 32, 50-64. DOI: 10.1111/bjdp.12021

Dumontheil, I., Burgess, P., \& Blakemore, S.-J. (2008). Development of rostral prefrontal cortex and cognitive and behavioural disorders. Developmental Medicine \& Child Neurology, 50, 168-181. DOI: 10.1111/j.1469-8749.2008.02026.x

Ellefson, M., Shapiro, L., \& Chater, N. (2006). Asymmetrical switch costs in children. Cognitive Development, 21, 108-130. DOI: 10.1016/j.cogdev.2006.01.002

Fitzpatrick, C., McKinnon, R.D., Blair, C.B., \& Willoughby, M.T. (2014). Do preschool executive function skills explain the school readiness gap between advantaged and disadvantaged children? Learning and Instruction, 30, 25-31. DOI:

10.1016/j.learninstruc.2013.11.003 
Hughes, C. (2011). Changes and challenges in 20 years of research into the development of executive functions. Infant and Child Development, 20, 251-271. DOI: 10.1002/icd.736

Hughes, C., Devine, R.T., Ensor, R., Koyasu, M., Mizokawa, A., \& Lecce, S. (2014). Lost in translation? Comparing British, Japanese and Italian children's theory-of-mind performance. Child Development Research, 2014, Article ID 893492, 1-10.. DOI: $10.1155 / 2014 / 893492$

Hughes, C., Devine, R.T., \& Wang, Z. (2015). Mind-mindedness and theory of mind: A twogeneration cross-cultural comparison of parent-child dyads in Hong Kong and the United Kingdom. Manuscript submitted for publication.

Hughes, C., Ensor, R., Wilson, A., \& Graham, A. (2010). Tracking executive function across the transition to school: A latent variable approach. Developmental Neuropsychology, 35, 20-36. DOI: 10.1080/87565640903325691

Hughes, C., Roman, G., \& Ensor, R. (2014). Parenting and executive functions: Positive and negative influences. In C.L. Cooper (Ed.), Wellbeing: A complete reference guide, Volume 1. Wellbeing in chidlhood, Part 2. Parenting and chidlren's development (8:125). Oxford, UK: John Wiley \& Sons.

Hughes, C., Roman, G., Hart, M.J., \& Ensor, R. (2013). Does maternal depression predict young children's executive function? - A 4-year longitudinal study. Journal of Child Psychology and Psychiatry, 54, 169-177. DoI: 10.1111/jcpp.12014

Imada, T., Carlson, S.M., \& Itakura, S. (2013). East-West cultural differences in contextsensitivity are evident in early childhood. Developmental Science, 16, 198-208. DOI: 10.1111/desc. 12016 
Imbo, I., \& LeFevre, J.-A. (2009). Cultural differences in complex addition: Efficient Chinese versus adaptive Belgians and Canadians. Journal of Experimental Psychology: Learning Memory and Cognition, 35, 1465-1476. DOI: 10.1037/a0017022

Kudo, M.F., Lussier, C.M., \& Swanson, H.L. (2015). Reading disabilities in children: A selective meta-analysis of the cognitive literature. Research in Developmental Disabilities, 40, 51-62. DOI: 10.1016/j.ridd.2015.01.002

Kuwabara, M., \& Smith, L.B. (2012). Cross-cultural differences in cognitive development: Attention to relations and objects. Journal of Experimental Child Psychology, 113, 2035. DOI: 10.1016/j.jecp.2012.04.009

Lewis, C., Koyasu, M., Oh, S., Ogawa, A., Short, B., \& Huang, Z. (2009). Culture, executive function, and social understanding. In C. Lewis \& J. I. M. Carpendale (Eds.), Social interaction and the development of executive function. New Directions in Child and Adolescent Development, 123, 69-85. DOI: 10.1002/cd.236

Logan, G. (1994). On the ability to inhibit thought and action: A users' guide to the stop signal paradigm. In D. Dagenbach \& T. H. Carr (Eds.), Inhibitory processes in attention, memory, and language (pp. 189-239). San Diego, CA US: Academic Press.

Miyake, A., Friedman, N. P., Emerson, M. J., Witzki, A. H., Howerter, A., \& Wager, T. D. (2000). The unity and diversity of executive functions and their contributions to complex "frontal lobe" tasks: A latent variable analysis. Cognitive Psychology, 41, 49-100. DOI: 10.1037/0012-1649.44.2.575

Nisbett, R.E., Choi, I., Peng, K., \& Norenzayan, A. (2001). Culture and systems of thought: Holistic versus analytic cognition. Psychological Review, 108, 291-301. DOI: 
10.1037//0033-295X.108.2.291

Reimers, S., \& Maylor, E. A. (2005). Task switching across the life span: Effects of age on general and specific switch costs. Developmental Psychology, 41, 661-671. DOI: $10.1037 / 0012-1649.41 .4 .661$

Rogers, R. D., \& Monsell, S. (1995). Costs of a predictable switch between simple cognitive tasks. Journal of Experimental Psychology: General, 124, 207-231.

Sabbagh, M.A., Xu, F., Carlson, S.M., Moses, L.J., \& Lee, K. (2006). The development of executive functioning and theory of mind: A comparison of Chinese and U.S. preschoolers. Psychological Science, 17, 74-81. DOI: 10.1111/j.1467$9280.2005 .01667 . x$

Schoemaker, K., Mulder, H., \& Deković, M. 2013. Executive Functions in Preschool Children with Externalizing Behavior Problems: A Meta-Analysis. Journal of Abnormal Child Psychology, 41, 457-471. DOI: 10.1007/s10802-012-9684-x

Tardif, T., Wang, L., \& Olson, S. L. (2009). Culture and the development of regulatory competence: Chinese-U.S. comparisons. In S. L. Olson \& A. J. Sameroff (Eds.), Biopsychosocial regulatory processes in the development of childhood behavior problems (pp. 258-289). New York: Cambridge University Press.

DOI:10.1017/CBO9780511575877.012

Wang, Z., Devine, R.T., Wong, K.K., \& Hughes, C. (2016). Theory of mind and executive function in middle childhood across cultures. Journal of Experimental Child Psychology, 149, 6-22. DOI: 10.1016/j.jecp.2015.09.028

Welsh, M (1991). Rule-guided behavior and self-monitoring on the tower of Hanoi disk- 
transfer task. Cognitive Development, 6, 59-76.

Wiebe, S.A., Espy, K.A., \& Charak, D. (2008). Using confirmatory factor analysis to understand executive control in preschool children: I. Latent structure. Developmental Psychology, 44, 575-587. DOI: 10.1037/0012-1649.44.2.575

Wu, S., \& Keysar, B. (2007). The effect of culture on perspective taking. Psychological Science, 18, 600-606. DOI: 10.1111/j.1467-9280.2007.01946.x

Wu, S., Barr, D.J., Gann, T.M., \& Keysar, B. (2013). How culture influences perspective taking: differences in correction, not integration. Frontiers in Human Neuroscience, 7, 17, DOI: $10.3389 /$ fnhum.2013.00822 